Original paper

\title{
Impact of spherical applicator diameter on relative biologic effectiveness of low energy IORT X-rays: A hybrid Monte Carlo study
}

\author{
Reza Shamsabadi, Hamid Reza Baghani *, Behnam Azadegan, Ali Asghar Mowlavi \\ Physics Department, Hakim Sabzevari University, Daneshgah Blvd, P.O. 9617976487, Sabzevar, Iran
}

\section{A R T I C L E I N F O}

\section{Keywords:}

Low-kV IORT

Breast cancer

Spherical applicator

Relative biologic effectiveness

Hybrid Monte Carlo simulation

\begin{abstract}
A B S T R A C T
Introduction: Low-kV IORT (Low kilovoltage intraoperative radiotherapy) using INTRABEAM machine and dedicated spherical applicators is a candidate modality for breast cancer treatment. The current study aims to quantify the RBE (relative biologic effectiveness) variations of emitted X-rays from the surface of different spherical applicators and bare probe through a hybrid Monte Carlo (MC) simulation approach.

Materials and methods: A validated MC model of INTRABEAM machine and different applicator diameters, based on GEANT4 Toolkit, was employed for RBE evaluation. To doing so, scored X-ray energy spectra at the surface of each applicator diameter/bare probe were used to calculate the corresponding secondary electron energy spectra at various distances inside the water and breast tissue. Then, MCDS (Monte Carlo damage simulation) code was used to calculate the RBE values according to the calculated electron spectra.

Results: Presence of spherical applicators can increase the RBE of emitted X-rays from the bare probe by about $22.3 \%$. In return, changing the applicator diameter has a minimal impact (about 3.2\%) on RBE variation of emitted X-rays from each applicator surface. By increasing the distance from applicator surface, the RBE increments too, so that its value enhances by about $10 \%$ with moving from 2 to $10 \mathrm{~mm}$ distance. Calculated RBE values within the breast tissue were higher than those of water by about $4 \%$ maximum value.

Conclusion: Ball section of spherical IORT applicators can affect the RBE value of the emitted X-rays from INTRABEAM machine. Increased RBE of breast tissue can reduce the prescribed dose for breast irradiation if INTRABEAM machine has been calibrated inside the water.
\end{abstract}

\section{Introduction}

Intraoperative radiotherapy (IORT) via isotropic low-kV $\mathrm{X}$-rays, known as low-kV IORT, has been proposed as one of the candidate modalities for early-stage breast cancer treatment $[1,2]$. The physical and biological properties of low energy X-rays make them an ideal choice in clinical practice [2]. Irradiation of the tumor bed with low energy X-rays, through a single fraction session, delivers a high localized dose to the tumor bed immediately after the surgery. Hence, it can significantly improve the local tumor control probability. Moreover, the short treatment time and increased relative biologic effectiveness (RBE) compared to the high-energy photons beam are the other advantages of low-kV IORT modality [3]. These low energy X-rays can lead to locally concentrated ionizations which consequently increase the RBE value.

INTRABEAM machine (Carl Zeiss Meditec AG, Oberkochen, Germany) is a dedicated system that is widely employed for low-kV IORT purposes. This machine is equipped with a special probe for X-ray production (with nominal energy of $50 \mathrm{kV}$ ) and different applicator shapes which enables this system to treat different cancer types distributed at various anatomic areas [4]. The clinical efficacy of this machine for breast cancer treatment has been addressed in several studies $[3,5,6]$. Dedicated spherical applicators have been introduced to irradiate the remaining tumor bed after the breast-conserving surgery (BCS). The reason for employing this applicator geometry for breast irradiation is the semi-spherical anatomy of the lumpectomy cavity after the tumor resection and demanded conformity between the employed applicator surface and tumor bed.

To irradiate the breast tumor bed, a proper spherical applicator diameter fills the lumpectomy cavity and the X-ray probe is exactly located at the center of the employed applicator. Then, the tumor bed is isotropically irradiated by the emitted X-rays from the probe tip. The delivered dose to the breast in this treatment modality is equal to $20 \mathrm{~Gy}$ which is prescribed at the surface of the employed spherical applicator diameter $[7,8]$.

\footnotetext{
* Corresponding author.

E-mail address: hamidreza.baghani@gmail.com (H.R. Baghani).
} 
Although the photons are nearly always considered as the low linear energy transfer (LET) radiation, according to the Astore et al. study, the LET of low energy X-rays is similar to high LET radiations like neutron and heavy ions [9]. It has been proven that the severity of the DNA damage induced by the high LET radiations is substantially more than those created by low LET ones [10]. Therefore, an increased RBE would be observed in the case of these high LET radiations. As suggested by Nikjoo and Lindborg [11], the RBE of the low-kV X-ray is remarkably higher than unity and can sometimes reach to about 1.5, based on the biological conditions and physical beam characteristics.

The interaction of the ionizing radiations (low energy X-rays here) with cells causes various direct and indirect DNA damages. The corresponding mechanism is that the direct ionization of the DNA molecule or later generation of hydroxyl radicals and hydrated electrons at the proximity of the DNA cause some damages to the DNA molecular structure. The most common types of DNA damages can be categorized as base damage (BD), single-strand break (SSB), and double-strand break (DSB). The DSBs are responsible for the most fatal form of DNA damage caused by ionizing radiation. On the other hand, the SSB lesions can be rapidly and effectively repaired in the excision repair process [12,13]. Total absorbed dose magnitude, radiation energy distribution, cell type, and oxygen level can influence the type and distribution of induced DNA damages. The reactive oxygen species (ROS) in aerobic conditions have an increased tendency for interaction with DNA molecules which can increase the induced DSB damages. In return, the presence of hypoxic conditions will decrease the DSB yield by about $40 \%[14,15]$.

The RBE value for a test radiation is directly proportional to the induced DNA damages (both SSB and DSB damages) and would be increased by increment of DNA damages.

In our previous study, it was shown that the presence of spherical applicators around the bare probe can substantially change the energy spectrum of the emitted X-rays from the INTRABEAM X-ray source [16]. Moreover, it was demonstrated that the variations of the spherical applicator diameter can also affect the employed X-ray energy (emitted from the applicator surface) for breast irradiation. On the other hand, variations of the emitted X-rays from the applicator surface consequently influence the energy distribution of produced secondary electron by photon interactions inside the medium. Hence, the linear energy transfer (LET) of secondary electrons can change with the variations of employed spherical applicator diameter. These variations in LET of secondary released electrons can finally change the RBE value for emitted X-rays from the surface of different spherical applicator diameters. These RBE variations may cause changing the prescribed dose when different applicator diameters are used for intraoperative breast irradiation by the INTRABEAM machine.

Although the variations of X-ray physical characteristics including the mean and most probable energy with changing the applicator diameter were fully evaluated in our previous study (through scoring the emitted X-ray energy spectrum from the surface of different spherical applicator diameters), no quantitative assessment about the influence of these X-ray energy variations on radiobiological features of low energy X-ray IORT (e.g. the RBE value) have not been presented at the mentioned work. Therefore, the current study aims to quantitatively evaluate the variations of the X-ray RBE by changing the spherical applicator diameter. To doing so, a hybrid simulation by GEANT4/ MCDS Monte Carlo codes was performed and RBE values for different applicator diameters as well as the bare probe itself were obtained inside the water and breast tissue. In this regard, a validated Monte Carlo model of INTRABEAM machine along with different applicator diameters, based on GEANT4 MC Toolkit, was used to obtain the energy spectrum of released secondary electrons within the studied media and then the obtained electron spectra were imported to the MCDS MC code, as input files, to compute the RBE values.

\section{Material and methods}

2.1. MC simulation of released secondary electrons around the probe and different spherical applicator diameters

A previously validated Monte Carlo model of the INTRABEAM machine, based on the GEANT4v10.2p02 MC Toolkit, was employed at the current study [16] for calculation of secondary electron energy spectra. The INTRABEAM machine is equipped with a miniature X-ray source (Xray probe) which can produce the X-ray energy with nominal energies of 30,40 , and $50 \mathrm{kV}$. Nevertheless, only the greatest photon energy $(50 \mathrm{kV})$ is used for clinical purposes. Accordingly, the $50 \mathrm{kV} \mathrm{X}$-ray was also considered in the current study.

As previously mentioned, due to the semi-spherical anatomy of the lumpectomy cavity after the breast-conserving surgery, the INTRABEAM machine utilizes some dedicated spherical applicators with different diameters, ranging from 1.5 to $5 \mathrm{~cm}$ with $0.5 \mathrm{~cm}$ increments, to irradiate the tumor bed. Each applicator is made of a biocompatible polyetherimide material, known as Ultem. An additional Aluminum filter layer is embedded inside the small applicators $(\leq 3 \mathrm{~cm})$ to balance the beam hardening level and also protect the applicator shaft from the excess leakage. In greater applicator $(\geq 3.5 \mathrm{~cm})$ the thickness of the material creates sufficient beam hardening, so the Aluminum layer is eliminated in the construction of these big applicators [17]. All of the spherical applicator diameters and the bare probe alone were considered at this work.

To calculate the energy spectrum of the released secondary electrons at different distances from the bare probe and/or applicator surface, separate simulations were performed. In this regard, at first the X-ray energy spectra at the surface of the bare probe and different spherical applicator diameters were scored through employing the validated Monte Carlo model of the INTRABEAM machine along with its spherical applicators. Then, the calculated X-ray spectra were modeled as the isotropic point sources located at the center of a simple spherical geometry and emitted X-rays as well as their interactions within the medium were fully tracked to calculate the energy spectrum of released secondary electrons at the distances of 2, 5, 7 and $10 \mathrm{~mm}$. The radius of the global sphere around the source was equal to $10 \mathrm{~cm}$ to fully satisfy the photon and electron backscattering condition. The electron energy spectra calculations were performed inside both water and breast tissues. The composition of the breast tissue was taken from ICRU-46 report including $\mathrm{H}(10.6 \%), \mathrm{C}(33.2 \%), \mathrm{N}(3 \%), \mathrm{O}(52.7 \%), \mathrm{Na}$ (0.1\%), P (0.1\%), S (0.2\%) and $\mathrm{Cl}(0.1 \%)$ with the density of $1.02 \mathrm{~g} / \mathrm{cm}^{3}$ [18]. It should be mentioned that the elemental composition of the breast tissue has been reported by mass fraction.

An important concern during the radiation transport is that all interactions of the photon which result in producing the secondary electrons should be accurately modeled and very low energy electrons (such as Auger electrons) must be also taken into account because these low energy electrons have a great contribution in RBE value. Therefore, the G4Livermore physics list of GEANT4 (a low energy electromagnetic one which can transport the electrons and photons in the energy region of near-zero to $100 \mathrm{MeV}$ [19]) was used in the performed simulations to consider all photon interactions including the photoelectric effect, Compton scattering, Rayleigh scattering and their subsequent low energy electrons such as Auger electrons, Compton electrons, and photoelectrons.

Regarding the fact that the emitted gamma rays by the ${ }^{60}$ Co source were considered as the reference radiation for RBE calculation, it was also necessary to score the secondary electron spectrum resulted from the ${ }^{60} \mathrm{Co}$ source. It should be noted that the RBE value is sensitive to the selected reference radiation. Although the ${ }^{60}$ Co source has been widely used as reference radiation in radiobiological assessments, other source types such as (200 keV X-ray, ${ }^{192} \mathrm{I}$, and ${ }^{137} \mathrm{Cs}$ ) have been also employed as reference radiation by some literatures $[20,21]$.

The recommended irradiation setup by Friedland et al. [22] was 


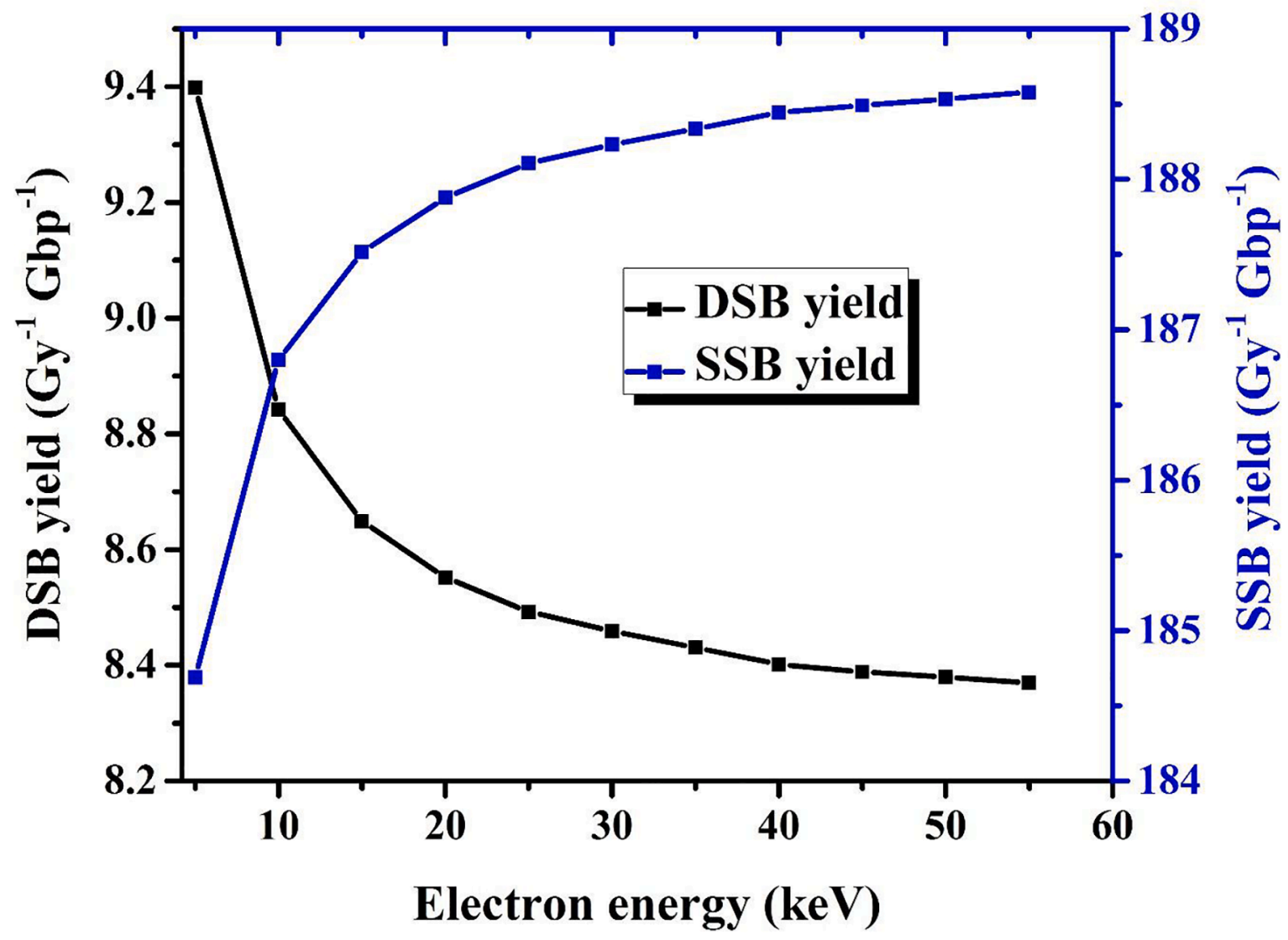

Fig. 1. Variations of DSB and SSB yields as function of electron energy, calculated by MCDS MC Code.

employed for scoring the released secondary electrons by the ${ }^{60} \mathrm{Co}$ source. Accordingly, a broad parallel beam of 1.17 and $1.33 \mathrm{MeV}$ photon energies was tracked inside a single-cell geometry and secondary electron were scored at the lower surface of the considered cell. The structure of this cell was simulated as a cylinder with the diameter and height of $20 \mu \mathrm{m}$. Moreover, an additional Mylar foil $\left(1.4 \mathrm{~g} / \mathrm{cm}^{3}\right.$ density) with the thickness of $4 \mu \mathrm{m}$ was assumed at the upper surface of the single-cell where the incident photons encounter with the cell. The calculated electron energy spectrum for the ${ }^{60} \mathrm{Co}$ source in the current study was identical to the reported one by Hsiao and Stewart [23] which had been stimulated by PENELOPE MC code.

It should be mentioned that 200 million particles were followed in each simulation to reduce the statistical uncertainty to less than $1 \%$. Furthermore, all of the simulations were performed by an Intel Core i7/8 GB RAM personal computer.

\subsection{Monte Carlo damage simulation (MCDS) and RBE calculation}

Since experimental determination of RBE value usually suffer from the large uncertainties, the MC method can be considered as an alternative approach for RBE evaluation [24]. MCDS (Monte Carlo Damage Simulation) code is a fast and dedicated micro-dosimetric code that can be employed for DNA damage simulation and RBE calculation for various particle types at a wide range of energies. The MCDS code provides very fast beneficial information to investigate the multi-scale effects and interactions of ionizing radiation in cells and allows rapid simulation of clustered DNA lesion distributions such as SSB, DSB, base damage, and etc., from which the RBE value for different radiations can be obtained.

The MCDS is not considered as an event-by-event MC code for track structure simulation [15]. On the other hand, this code uses four parameters including $\sigma_{S b}, f, N_{\min }$, and $\mathrm{n}_{\text {seg }}$ for DNA damage calculation which their values have been previously optimized by Semenenko and Stewart [25]. In this regard, at first, some number of elementary lesions (including either stand break (SB) or base damage (BD)) are randomly distributed along both strands of a DNA segment (in terms of base pairs (bps) in a cell per Gy of radiation). The length of the DNA segment is a function of $n_{\text {seg }}$. This parameter depends on the particle type and energy and can be expressed by the following Equation:

$n_{\text {seg }}(x)=149200-\frac{123600(x)}{x+267}$

where the $\mathrm{x}$ parameter can be defined by Eq. (2):

$x=\left(1-\frac{1}{1+\frac{T}{m_{0} c^{2}}}\right)^{-1}$

Here, $\mathrm{T}$ and $\mathrm{m}_{0} \mathrm{c}^{2}$ are the kinetic energy and the rest mass energy of the electron, respectively [26].

Then, these elementary lesions are divided into some classified clusters to determine the type of induced damages in the DNA segment $[25,26]$. Since the DNA lesions generated by ionizing radiation typically consist of base damage (BD) and stand break (SB), all types of DNA damages in the MCDS program can be classified into DSBs, SSBs, and BDs. Grouping the initial DNA lesions to separate clusters is specified by the $N_{\min }$ parameter. $N_{\min }$ is the minimum length of the undamaged base pairs between neighboring lesions and its optimum value during the DNA damage simulations is equal to $9 \mathrm{bps}$. A cluster contains all lesions (both BDs and SBs) which are distributed within the DNA length of $N_{\text {min }}$ (9 bps). After all lesions have been grouped to separate clusters, they would be further categorized as the SSBs, DSBs, and BDs. A cluster would be marked as the DSB if two neighbor SBs on the opposite DNA strands have been distributed within the $10 \mathrm{bp}$ length. On the other 
hand, all clusters which contain at least one SB but would not meet the above-mentioned criteria are labeled as the SSBs. The remaining clusters would be categorized as the BDs $[25,27,28]$. The number of BDs respect to the SBs in each performed DNA damage simulation would be controlled by $\sigma_{S b}$ and $f$ parameters which their optimum values during the DNA damage simulation would be equal to $1300 \mathrm{~Gy}^{-1}$ cell $^{-1}$ and 3, respectively. The $\sigma_{S b}$ parameter is defined as the number of individual strand breaks $\left(\mathrm{Gy}^{-1}\right.$ cell $\left.^{-1}\right)$, while $f$ is the ratio of the base damages to strand breaks. It should be mentioned that $\sigma_{S b}, f, N_{\min }$ are not dependent on the particle type and its kinetic energy [27].

DNA damage calculation by MCDS code can be performed in different oxygenation levels (hypoxic or normoxic conditions) [27]. The formation of strand breaks and base damages are also parameterized by an energy threshold, wherein the larger energy deposition than the threshold energy will generate a DNA lesion, whereas smaller energy deposition does not produce any DNA lesion [29]. The minimum energy threshold for DNA damage induction by electrons in the MCDS code is about $25.6 \mathrm{eV}$ and this threshold value increases for the heavy ions.

The accuracy of the MCDS code in DNA damage simulation and RBE estimation has been previously confirmed through conducted studies by Stewart and colleagues $[23,25,26,27,30]$.

The SSB and DSB yields are mostly dependent on the particle energy. The plot of SSB and DSB yield as a function of electron energy below 50 $\mathrm{keV}$, obtained by MCDS code, is illustrated in Fig. 1. As demonstrated in this Figure, the DSB yield decrease with the increment of electron energy, while a reverse behavior would be observed for SSB yield with increasing the electron energy. This finding is similar to the previously published results by Reniers et al. study [31].

The MCDS 3.00B version was employed at the current study for DNA damage simulation and RBE estimation for emitted low energy X-rays from the surface of the different spherical IORT applicators and the bare probe. It should be mentioned that a wide variety of charged particles (such as electron, proton, alpha, ${ }^{20} \mathrm{Ne},{ }^{16} \mathrm{O}$ and etc.) have been considered in this version of MCDS code for RBE evaluation [27,25,23]. Nevertheless, direct determination of damage yields for photons is not provided [23]. In return, the initial energy spectrum of secondary electrons released by photon interactions inside the interested target volume (water and breast tissue in the current study) should be given to this code in order to compute the induced DNA damages and corresponding RBE values for photon beam.

The scored electron energy spectra at various distances $(2,5,7$, and $10 \mathrm{~mm}$ ) from the surface of different spherical applicator diameters and bare probe, within the water and breast tissue, were imported into the MCDS code and strand break yield $\left(\mathrm{Gy}^{-1} \mathrm{Gbp}^{-1}\right)$ was calculated. The reference strand break yield $\left(\mathrm{Gy}^{-1} \mathrm{Gbp}^{-1}\right)$ was also obtained by importing the reference secondary electron energy spectrum, resulted from the ${ }^{60}$ Co source within the single-cell geometry, into the MCDS code. Owing to the fact that the SSBs are considered as the sublethal damages, only the DSB yields were considered for RBE calculation. Accordingly, the RBE values for emitted X-rays from the surface of different spherical applicator diameters as well as the bare probe alone were obtained through the following Equation [32]:

$R B E=\frac{(\text { strand break yield })}{(\text { strand break yield })_{\text {reference }}}$

In which the strand break yield is attributed to the DSB yield for either reference or test radiation (low-kV IORT X-rays). It should be noted that the DSB yield for reference radiation was obtained as 8.28 which is in accordance with the reported value by other literatures $[23,31,33]$.

It is worth mentioning that the RBE evaluations were performed under the fully aerobic condition $\left(\mathrm{pO}_{2}=100 \%\right)$.

To justify the trend of RBE variations, the obtained electron energy spectra at different distances from the surface of different spherical IORT applicators and/or the bare probe were employed.

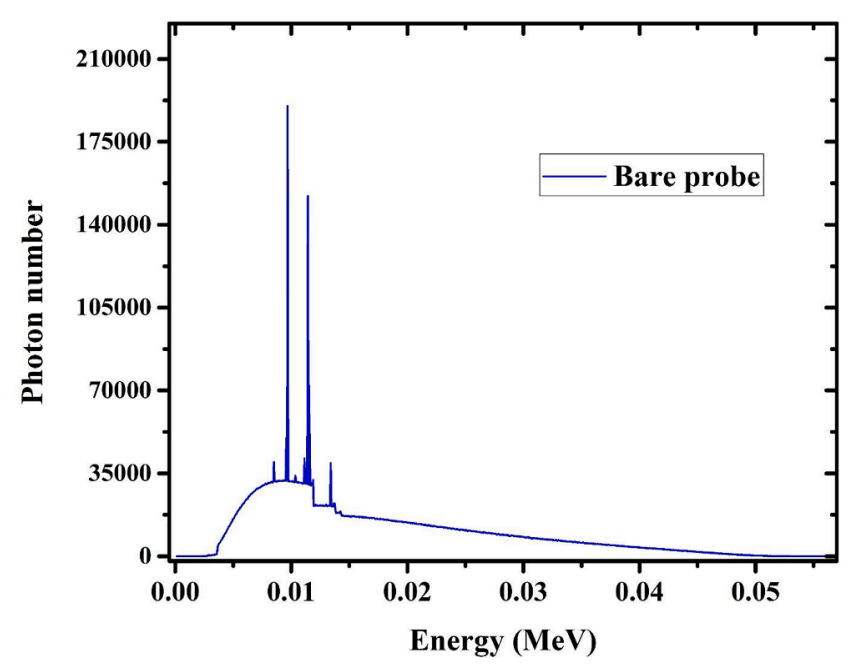

Fig. 2. Energy spectrum of emitted X-rays from the surface of the bare probe at $50 \mathrm{kV}$ nominal accelerating potential.

\section{Results and discussion}

The emitted X-ray energy spectrum from the surface of the bare probe at $50 \mathrm{kV}$ nominal energy is depicted in Fig. 2. As illustrated in this Figure, the spectrum consists of bremsstrahlung photons (presented continuum) and characteristic X-rays (presented peaks) which are mainly produced by K-shell relaxation processes.

It has been previously shown that the presence of the spherical applicator around the bare probe can remarkably alter the shape of the X-ray spectrum [16] and consequently may affect the X-ray RBE value through changing the secondary electron energy spectrum.

\subsection{MC calculated secondary electron spectra}

The scored secondary electron spectra inside the breast tissue at various distances of $2,5,7$, and $10 \mathrm{~mm}$ from the surface of the bare probe, are depicted in Fig. 3.

As demonstrated in Fig. 3, the contribution of the secondary electrons produced from different mechanisms including photoelectric, Compton, Auger interactions, as well as the total secondary electron spectra are shown separately. As depicted in Fig. 3, the contribution of Auger electrons in total spectrum increments by increasing the depth within the breast tissue, so that the Auger electron peak increases by about 2.1 times with moving from $2 \mathrm{~mm}$ to $10 \mathrm{~mm}$ depth from the surface of the bare probe. This fact is mainly because of increasing the photoelectric interactions with increment of the depth within the breast tissue which can finally lead to the increment of Auger electron intensity. Furthermore, as it is obvious in Fig. 3, the intensity of photoelectrons is much higher than the Compton electrons which would be linked to the low energy nature of the employed X-ray beam and higher cross-section of photoelectric effect relative to the Compton scattering in such low energy regions. The observed peaks at about 10, 11.4, and 13.4 $\mathrm{keV}$ are related to the released secondary electrons by the corresponding characteristics X-rays which are shown in Fig. 2.

The secondary electron spectra related to the $50 \mathrm{kV}$ X-rays (produced by Xsoft/Axxent electronic brachytherapy system) has been also reported by Reniers et al. through Monte Carlo simulation with EGSnrc MC code [31]. Since electron energies lower than $1 \mathrm{keV}$ cannot be transported by EGSnrc code, the contribution of Auger electrons (mainly from $\mathrm{O}, \mathrm{N}$, and $\mathrm{C}$ which with energies less than $1 \mathrm{keV}$ ), was tuned manually by assuming one Auger electron for each photoelectric interaction. The same approach was also employed for quantification of Auger electron intensities in White et al. study [34]. In a performed study by Ezzati for RBE evaluation in high energy clinical photon fields [35], the 

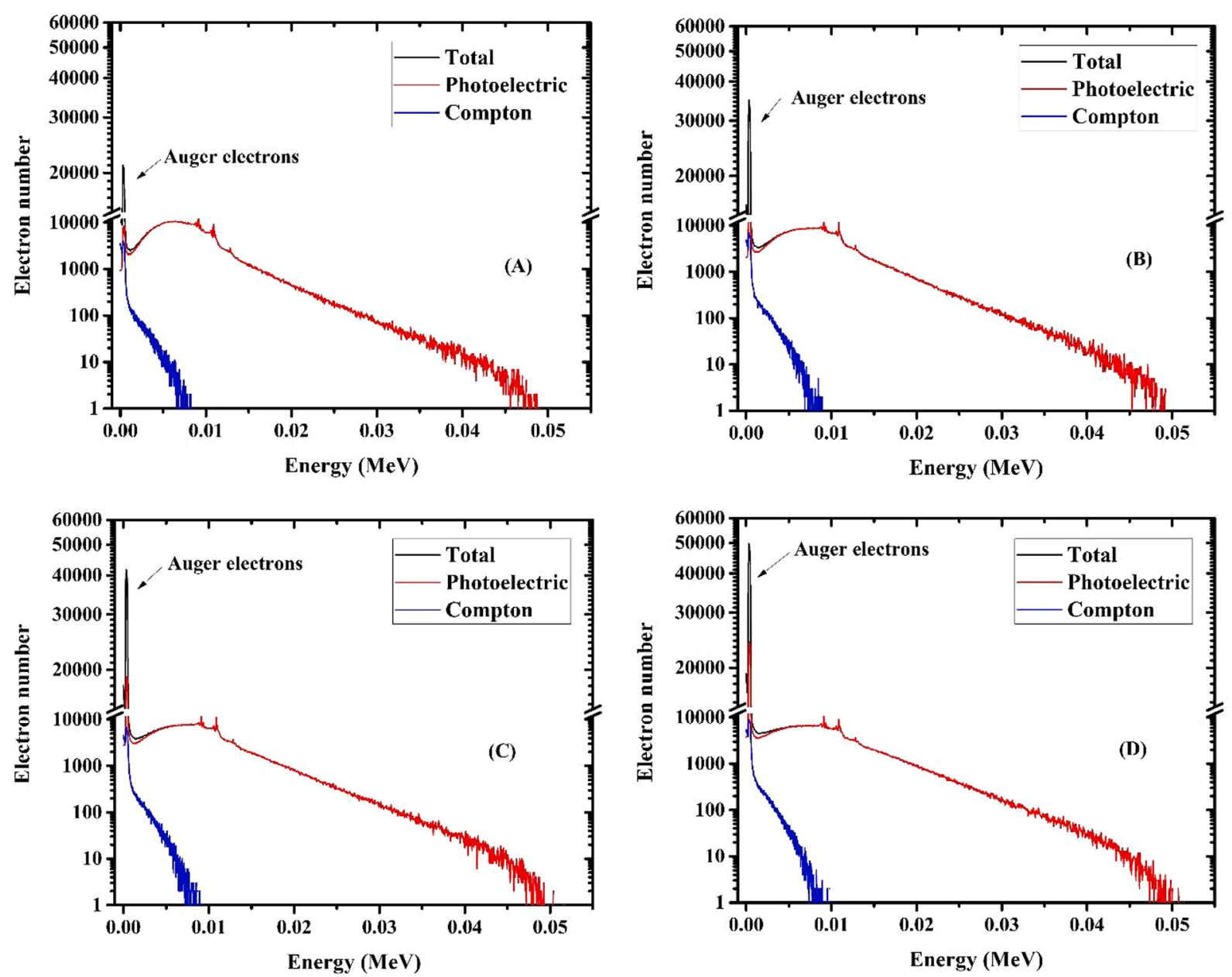

Fig. 3. Calculated secondary electron energy spectrum inside the breast tissue at various distances of (A) $2 \mathrm{~mm}$, (B) $5 \mathrm{~mm}$, (C) $7 \mathrm{~mm}$, and (D) $10 \mathrm{~mm}$ from the bare probe surface. 
contribution of the secondary electrons with energies lower than $2 \mathrm{keV}$ in the total spectrum has been estimated by Vassiliev calculations [36] and no direct electron tracking has been done in this regard. This fact was also due to employing the MCNPX MC code in the mentioned study which could not transport the electron beam at energies lower than 1 $\mathrm{keV}$. On the other hand, the produced low energy Auger electrons were fully obtained through direct electron transportation by GEANT4 MC Toolkit at the current study.

Calculated secondary electron energy spectra at various distances of $2,5,7$, and $10 \mathrm{~mm}$ from the surface of 1.5 and $5 \mathrm{~cm}$ spherical applicator diameters (the smallest and greatest applicator diameters), inside the breast tissue, have been presented in Figs. 4 and 5, respectively. Due to the huge number of such obtained results, the secondary electron spectra for the rest of the applicator diameters are not displayed. Nevertheless, it is emphasized that a similar trend was also observed for non-reported applicators.

In contrast to those obtained for the bare probe alone (shown in Fig. 3), small variations were observed in the intensity of the Auger electrons, Compton ones, and photoelectrons at different distances from the applicator surface, as depicted by Figs. 4 and 5. Consequently, the total electron spectra do not remarkably change with increasing the distance from the applicator surface.

A comparison between the presented results in Fig. 3 and those of Figs. 4 and 5 indicates that the shape of the secondary electron spectra
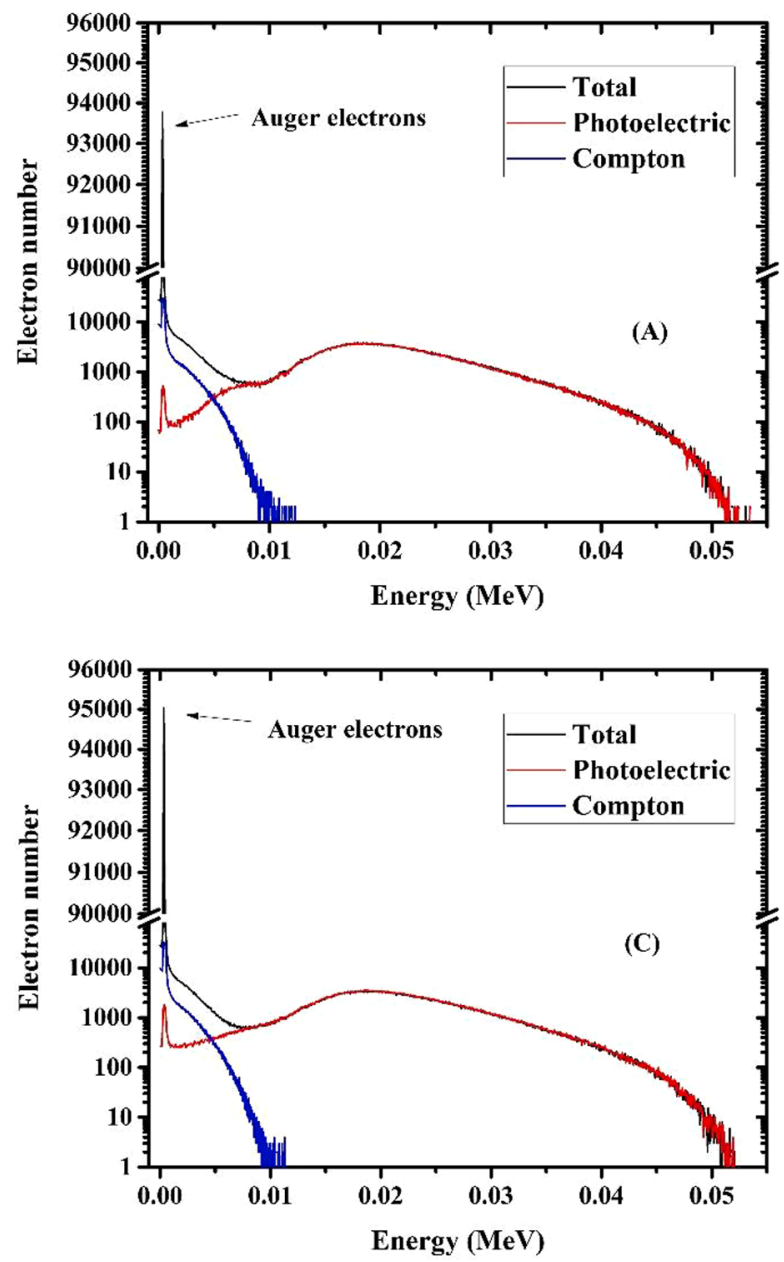

would substantially change in presence of spherical applicator. This finding is likely due to the induced beam hardening and attenuation by the ball section of the spherical applicator which can finally alter the Xray energy spectrum and as a consequence, change the energy spectrum of released secondary electrons. As demonstrated, the intensity of the Auger and Compton electrons in the total spectrum increase when the bare probe has been embedded with the spherical applicator, while the intensity of the photoelectrons decrements. Introduced beam hardening by the ball section of the spherical IORT applicator will increase the mean X-ray energy [16] and therefore, the probability of photoelectric interaction decreases. In return, the probability of Compton scattering would be increased, and hence, the intensity of Compton electron in the total electron energy spectrum increments as well. Increasing the contribution of low energy Compton electrons can also amplify the intensity of the consequent Auger electrons.

Besides, as shown in Figs. 4 and 5, the contribution of the released secondary electrons by characteristic X-rays in the total spectrum reduces to a negligible level when the applicator is inserted. This finding is also due to the significant filtration of the characteristic X-rays by the ball section of the spherical applicator. However, these peaks can be clearly seen in the case of the bare probe alone (as shown in Fig. 3).

Secondary electron spectra at various distances from the bare probe surface within the water are shown in Fig. 6.

As illustrated in Fig. 6, the obtained electron spectra inside the water
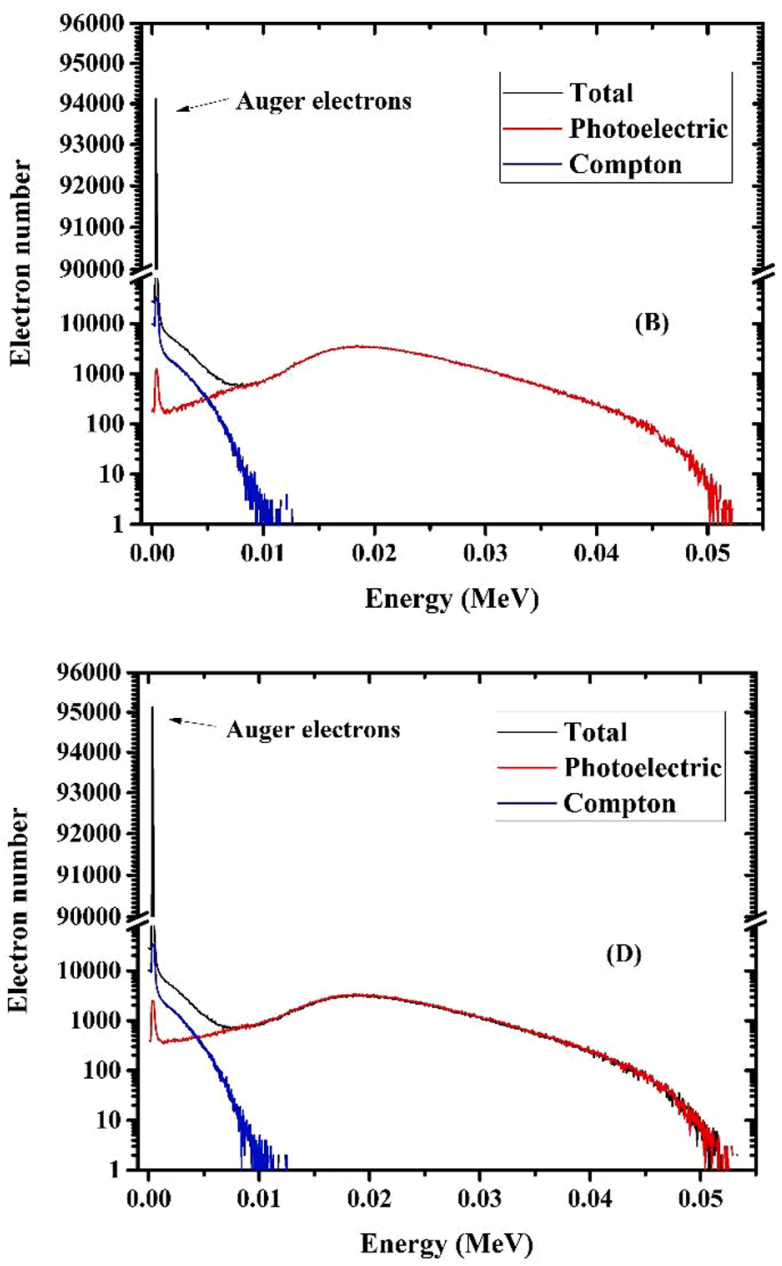

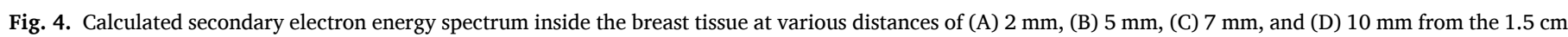
diameter applicator surface. 

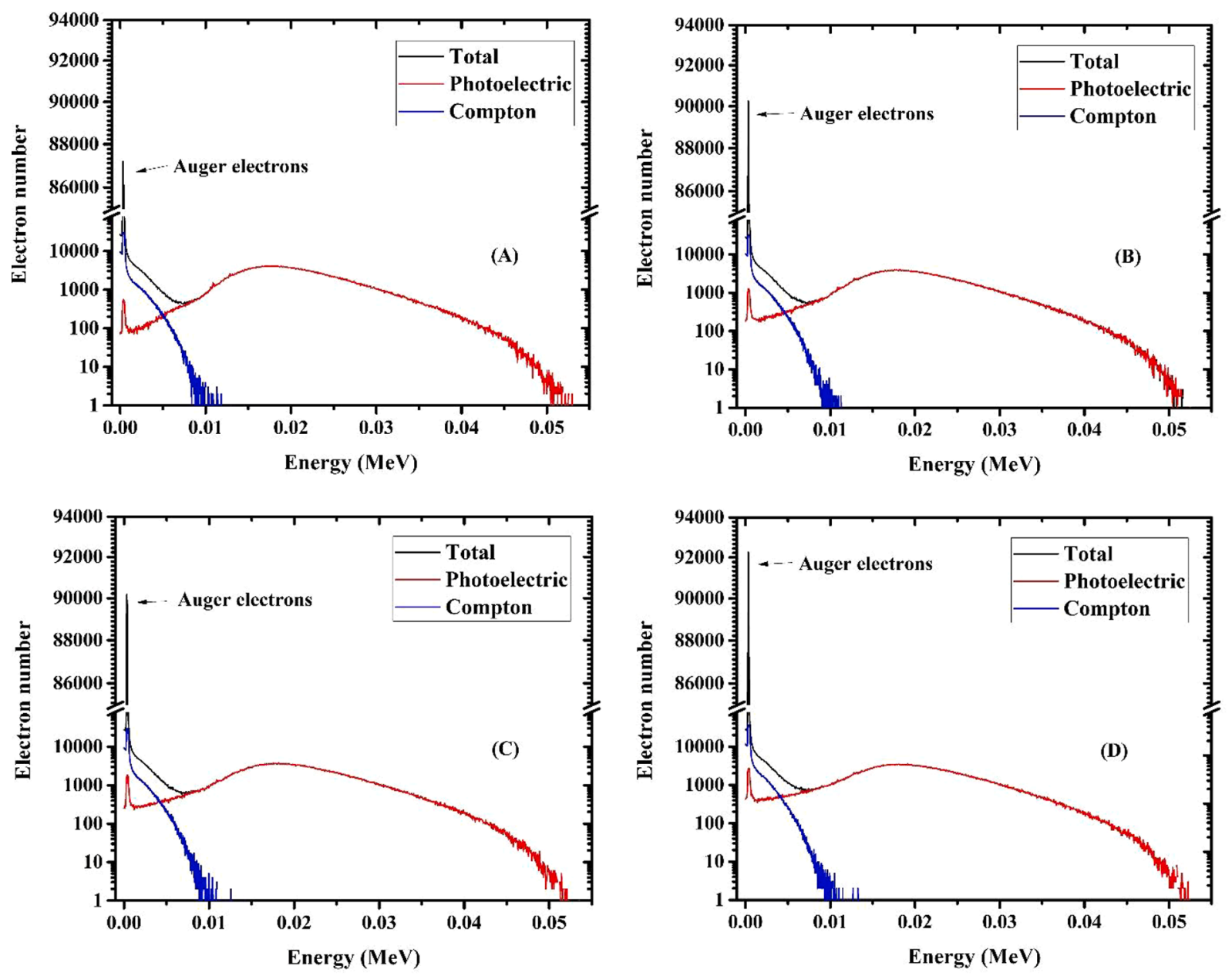

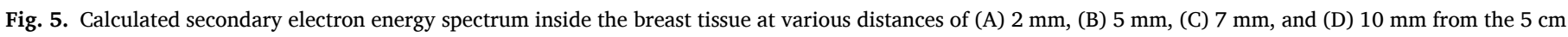
diameter applicator surface.

are analogous to those obtained within the breast tissue (as depicted in Fig. 3).

Calculated secondary electron spectra at various distances of $2,5,7$, and $10 \mathrm{~mm}$ from the surface of 1.5 and $5 \mathrm{~cm}$ spherical applicator diameters, inside the water, have been depicted in Figs. 7 and 8, respectively.

Comparison between the scored electron energy spectra inside the breast tissue and water reveals that the intensities of the Auger electrons within the breast tissue are more than relevant obtained values inside the water. This difference is about $11.1 \%$ and $9.5 \%$ at $10 \mathrm{~mm}$ distance from the surface of the brae probe and $5 \mathrm{~cm}$ diameter applicator, respectively. This finding is related to the presence of the greater atomic number constituents (such as $\mathrm{P}, \mathrm{S}, \mathrm{Cl}$, and $\mathrm{Na}$ ) in breast tissue composition as well as the higher density of the breast tissue respect to the water which can lead to the higher photon interaction probability (especially the photoelectric effect). As a result, the intensity of the consequently produced Auger electrons increases too. Nevertheless, a large number of Auger electrons are produced through photon interactions with both considered media. Totally, at the X-ray energy regions below $10 \mathrm{kV}$, the production of very low energy Compton electrons and Auger ones (below $1 \mathrm{keV}$ ) are more prominent [37]. This fact is obvious in the illustrated results in Figs. 7 and 8. These low energy electrons can cause increasing the RBE value of the low-kV IORT X-rays. The range of these low energy electrons (about $0.1 \mu \mathrm{m}$ ) is less than the cell size, and therefore, the produced Auger electrons inside the cell have a great influence on the DNA damage [38]. Accordingly, the presence of these low energy electrons can increase the RBE value. Contribution of the low energy electrons (mainly the Auger and very low energy Compton ones) in total electron spectra related to the bare probe and spherical applicator diameters of $1.5,3$, and $5 \mathrm{~cm}$ at different depths inside the water and breast tissue have been quantitatively reported in Table 1.

\section{2. $R B E$ evaluation for bare probe and different spherical applicator diameters}

To estimate the RBE value with the MCDS MC code, at first, the number of the DSB inductions, weighted by the intensities of the different electron energies inside the spectrum [25], was calculated for both low-kV IORT X-rays and reference radiation. Then, the corresponding RBE value was obtained by Eq. (3).

The variations of RBE value at different distances from the bare probe inside the water and breast tissue have been presented in Fig. 9.

As demonstrated in Fig. 9, the RBE value increments with increasing 

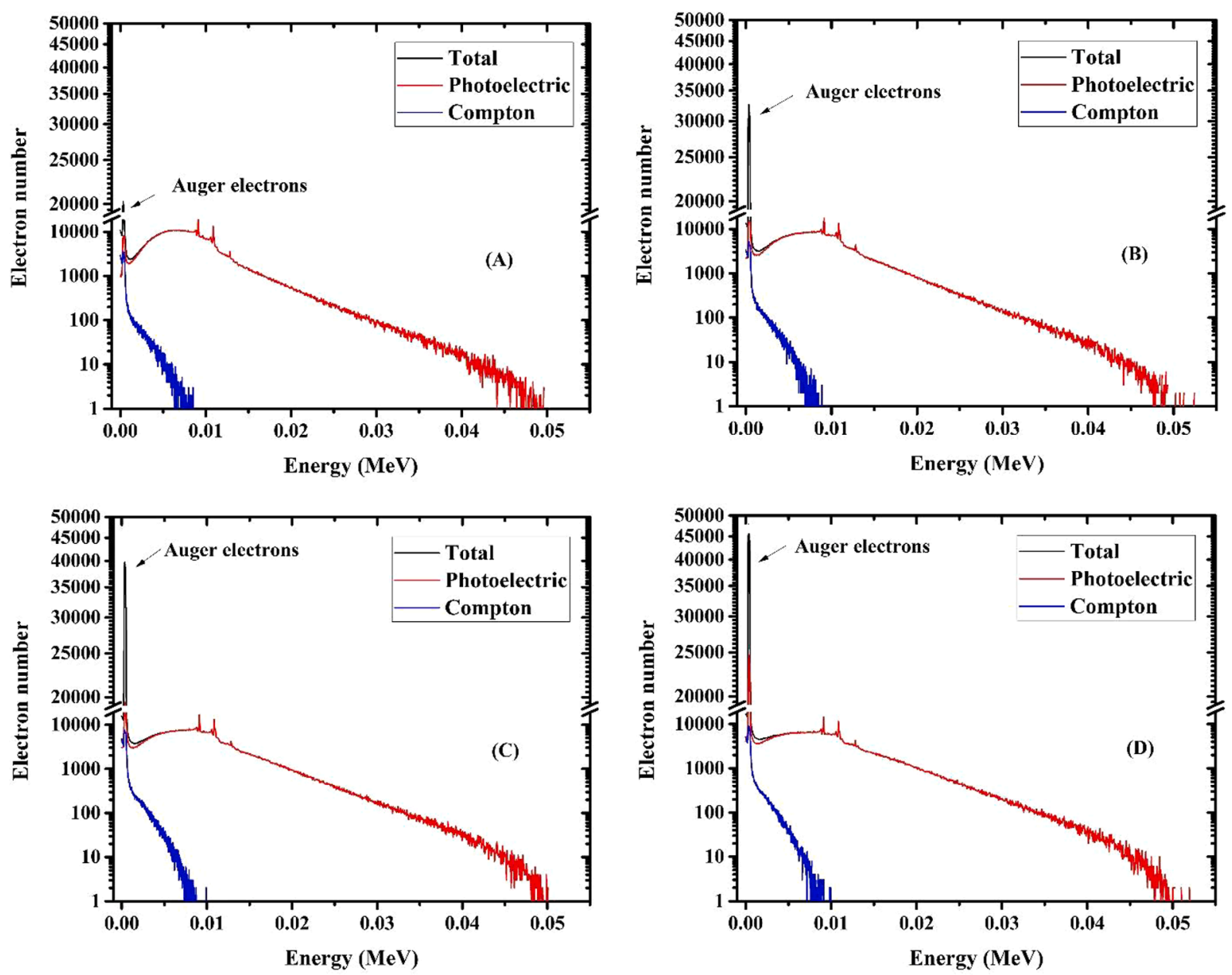

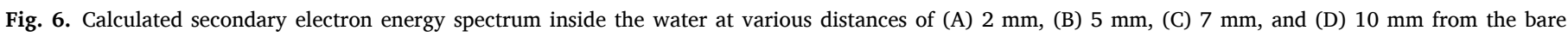
probe surface.

the depth inside the medium. This finding can be justified by the reported electron energy spectra in Figs. 3 and 6. As illustrated by these Figures, the intensity of the low energy electrons (below $1 \mathrm{keV}$ ) increments at greater distances from the probe surface. On the other hand, these low energy electrons have a considerable contribution to DSB induction (refer to Fig. 1). As a result, it can be expected that the RBE value will be increased at greater distances. The maximum RBE variation for both studied media was about $10 \%$.

The RBE value for the breast tissue varies from 1.23 at $2 \mathrm{~mm}$ distance from the bare probe surface to 1.36 at $10 \mathrm{~mm}$ distance, while these values range from 1.22 to 1.34 at corresponding distances inside the water. The higher RBE values for the breast tissue can be attributed to the higher number of the low energy electrons in the case of breast tissue respect to the water (refer to Table 1).

Variations of RBE value as a function of distance from the surface of different spherical applicator diameters inside the water and breast tissue are displayed in Fig. 10.

Generally, with increasing the applicator diameter from 1.5 to $3 \mathrm{~cm}$, the RBE value increments too. The maximum variation of RBE with changing the applicator diameter from 1.5 to $3 \mathrm{~cm}$ is about $1 \%$. The same trend is also observed for greater applicator diameters ( 3.5 to $5 \mathrm{~cm}$ ones), so that the RBE value increases by about $3.2 \%$ with an increment in applicator diameter from 3.5 to $5 \mathrm{~cm}$. The calculated RBE values for great applicator diameters $(\geq 3.5 \mathrm{~cm})$ are smaller than those calculated for small ones $(\leq 3 \mathrm{~cm})$ at the same distance. This fact may be related to the presence of Aluminum filter layer in the design of small applicators $(\leq 3 \mathrm{~cm})$ and its elimination in the construction of great ones $(\geq 3.5 \mathrm{~cm})$ which affects the intensity of the low energy electrons in total spectra.

From the obtained results, it can be deduced that changing the applicator diameter cannot substantially affect the RBE of the emitted Xrays from the surface of various applicator diameters (the maximum difference of $3.2 \%$ was observed in RBE value). Therefore, variations of employed spherical applicator diameter can not have a remarkable impact on the patient's prescribed dose, so that it can change by about $3 \%$ (which is equal to $0.6 \mathrm{~Gy}$ following the $20 \mathrm{~Gy}$ prescribed dose in breast low-kV IORT technique).

On the other hand, the presence of the spherical applicator can substantially change the RBE of the emitted X-rays from the surface of the bare probe alone. Comparison of the reported RBE values for the bare probe (Fig. 9) with those obtained for different applicator diameters (Fig. 10) indicates that the RBE value increments by the maximum amount of about $22.3 \%$ (in the case of $3 \mathrm{~cm}$ diameter applicator and $2 \mathrm{~mm}$ distance inside the breast tissue) when the spherical applicator envelops the bare probe. This finding is likely due to the increment of low energy electron contribution in total secondary electron spectra at the presence of spherical IORT applicator (refer to 

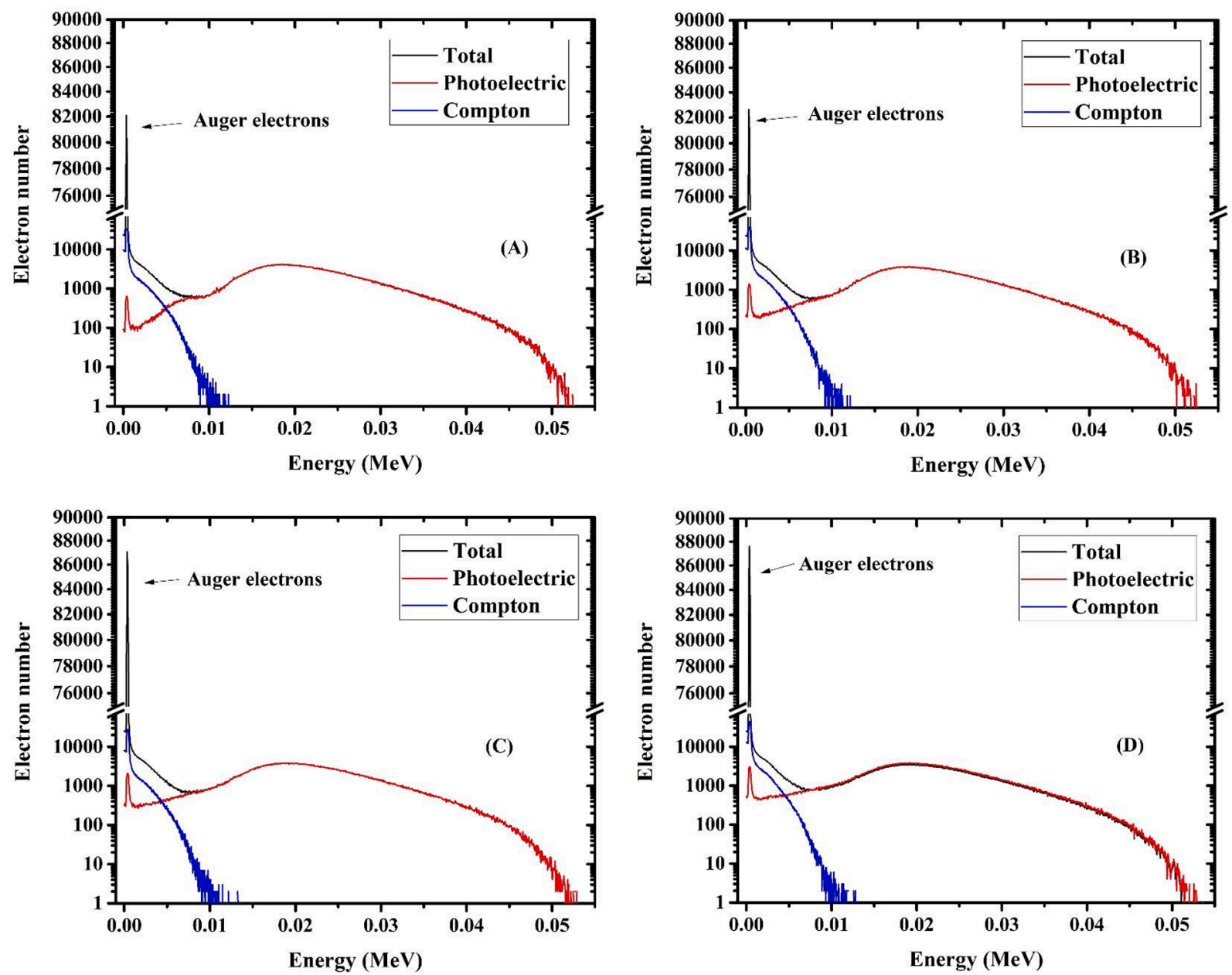

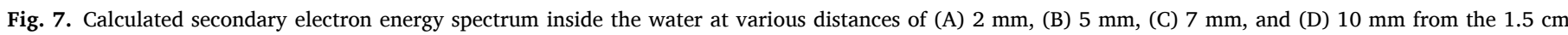
diameter applicator surface.

Table 1). For example, when the bare probe is embedded by the $3 \mathrm{~cm}$ diameter applicator diameter, the intensity of low energy electrons increases by about 3.6 times (at $2 \mathrm{~mm}$ distance from the surface). As a result, it can be expected that the RBE value enhances at the presence of the spherical IORT applicator.

The calculated RBE values inside the breast tissue, in presence of spherical applicators, are greater than those of water. This fact can also be justified by scored electron spectra within the water and the breast tissue as well as the reported data in Table 1. Comparison between the calculated electron spectra inside the breast tissue (Figs. 4 and 5) and those calculated within the water (Figs. 7 and 8) demonstrates that the intensity of low energy electrons is higher in the case of breast tissue (see also the reported data in Table 1). As a result, the RBE values would be greater for breast tissue. The most obvious difference in this regard is observed for $3 \mathrm{~cm}$ diameter spherical applicator, such that the RBE value increases by a maximum amount of $4 \%$ with moving from water to breast tissue (at $2 \mathrm{~mm}$ distance from the applicator surface). Since the INTRABEAM machine is calibrated inside the water, these RBE variations with moving from water to breast tissue can affect the prescribed dose. If the machine has been calibrated inside the water, prescription of 20 Gy dose is biologically equivalent to 20.8 Gy absorbed dose by the irradiated breast tissue. Therefore, the treatment team can reduce the prescribed dose by up to $4 \%$ during patient treatment planning and dose prescription.

Although the GEANT4 MC Toolkit has also the capability of DNA damage evaluation via the G4DNA physics list, we used the MCDS MC code for this purpose. Generally, applying the MCDS code for DNA damage estimation and RBE evaluation has some advantages over the direct GEANT4 simulation. Firstly, the MCDS code is a fast Monte Carlobased DNA damage simulator algorithm and needs less time to result in the intended radiobiological data in comparison with the GEANT4 MC Toolkit. Secondly, the MCDS code is more straight forward and userfriendly than the GEANT4 in the calculation of induced DNA damages by ionizing radiation, because all of the required algorithms for random lesion distribution along the considered DNA length and grouping the different DNA damage types have been automatically performed by the code itself, while these procedures should be personally developed through writing the relevant computational algorithms by the user in the case of GEANT4 MC Toolkit.

\section{Conclusion}

The influence of applicator diameter on RBE of employed low energy $\mathrm{X}$-rays for breast IORT purposes was quantitatively evaluated through a 

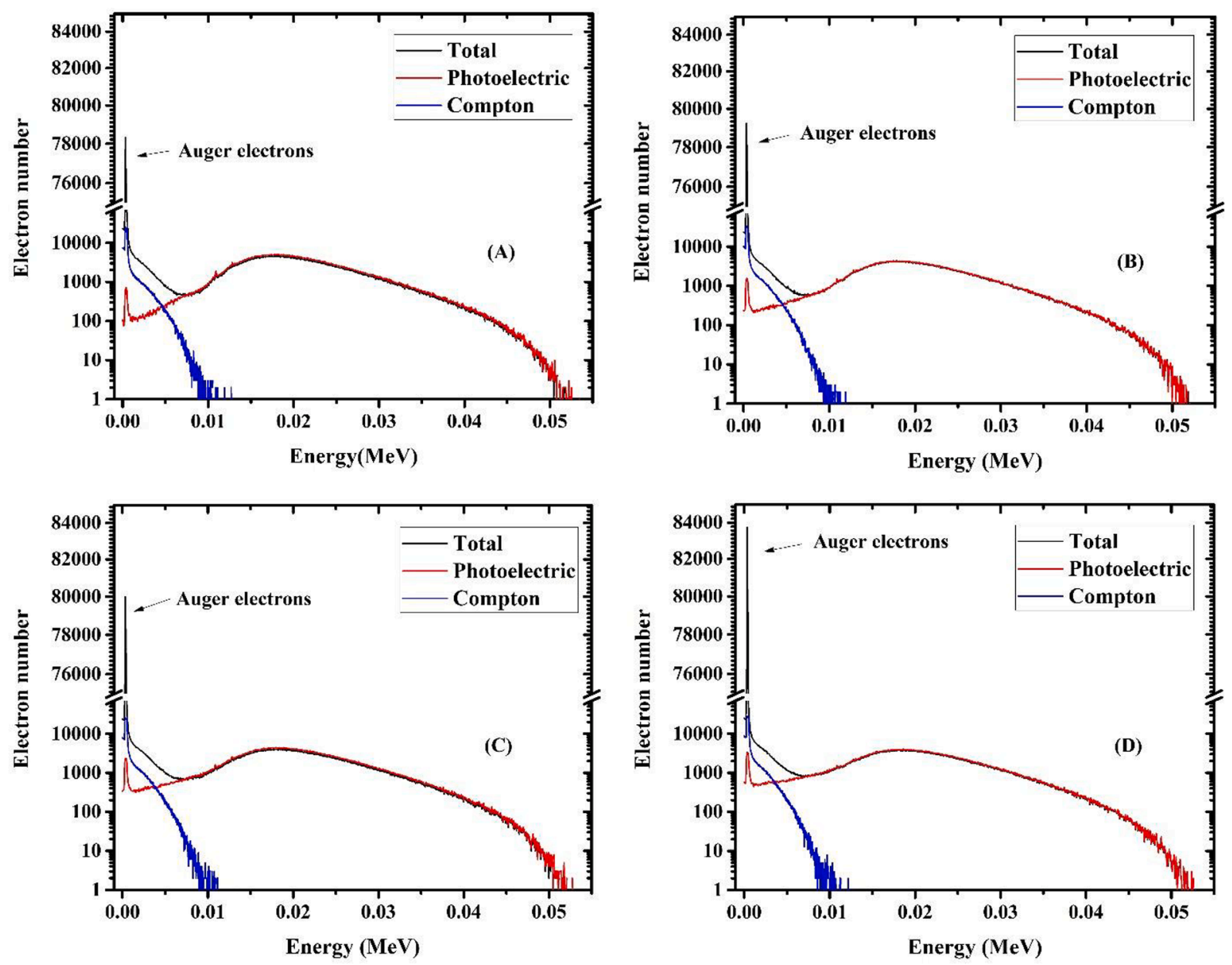

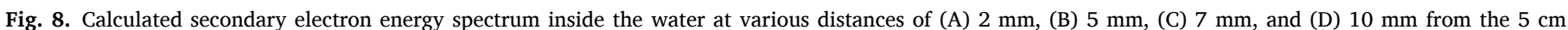
diameter applicator surface.

hybrid GEANT4/MCDS approach inside the water and breast tissue.

The results demonstrated that the presence of the spherical applicators can substantially change the RBE of the emitted X-rays from the surface of the bare probe alone (by about 22.3\%). Nevertheless, variations of applicator diameter have a minimal impact on the obtained RBE values (maximum level of 3.2\%).

Increasing the depth within the irradiated media can also increase the RBE value by about $10 \%$ in both water and breast tissue.

Obtained RBE values inside the breast tissue were somewhat higher (about $4 \%$ at the maximum level) than those calculated within the water. This RBE increment can a little affect the prescribed dose during the low-kV IORT of breast cancer.

Table 1

Contribution of low energy electrons (below $1 \mathrm{keV}$ ) in total electron energy spectra (in terms of percentage (\%)) for the bare probe and spherical applicator diameters of $1.5,3$, and $5 \mathrm{~cm}$ which have been calculated at different depths inside the water and breast tissue.

\begin{tabular}{|c|c|c|c|c|c|c|c|c|}
\hline \multirow{2}{*}{$\begin{array}{l}\text { App. diameter } \\
\text { (cm) }\end{array}$} & \multicolumn{4}{|c|}{ Depth in water (mm) } & \multicolumn{4}{|c|}{ Depth in breast tissue (mm) } \\
\hline & 2 & 5 & 7 & 10 & 2 & 5 & 7 & 10 \\
\hline Bare probe & 4.7 & 7.1 & 8.5 & 10.1 & 5.4 & 8.3 & 9.7 & 11.5 \\
\hline 1.5 & 16.6 & 16.8 & 17.7 & 17.8 & 18.9 & 19.0 & 19.4 & 19.5 \\
\hline 3 & 16.7 & 17.6 & 17.7 & 17.9 & 19.2 & 19.4 & 19.8 & 20.1 \\
\hline 5 & 15.8 & 16.2 & 16.3 & 17.0 & 17.8 & 18.4 & 18.5 & 18.8 \\
\hline
\end{tabular}

Totally, having enough knowledge about the RBE of the low energy $\mathrm{X}$-rays can help to improve the accuracy of dose prescription during the IORT procedure, where a more concern would be existed due to the single fraction nature of this treatment modality.

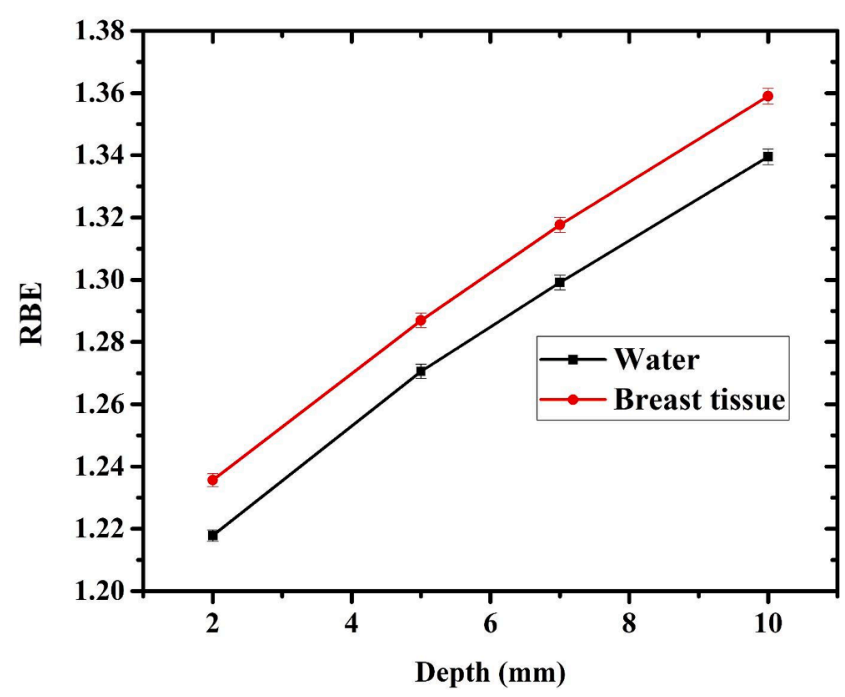

Fig. 9. Variations of RBE value as a function of depth inside the water and breast tissue for the bare probe alone. 

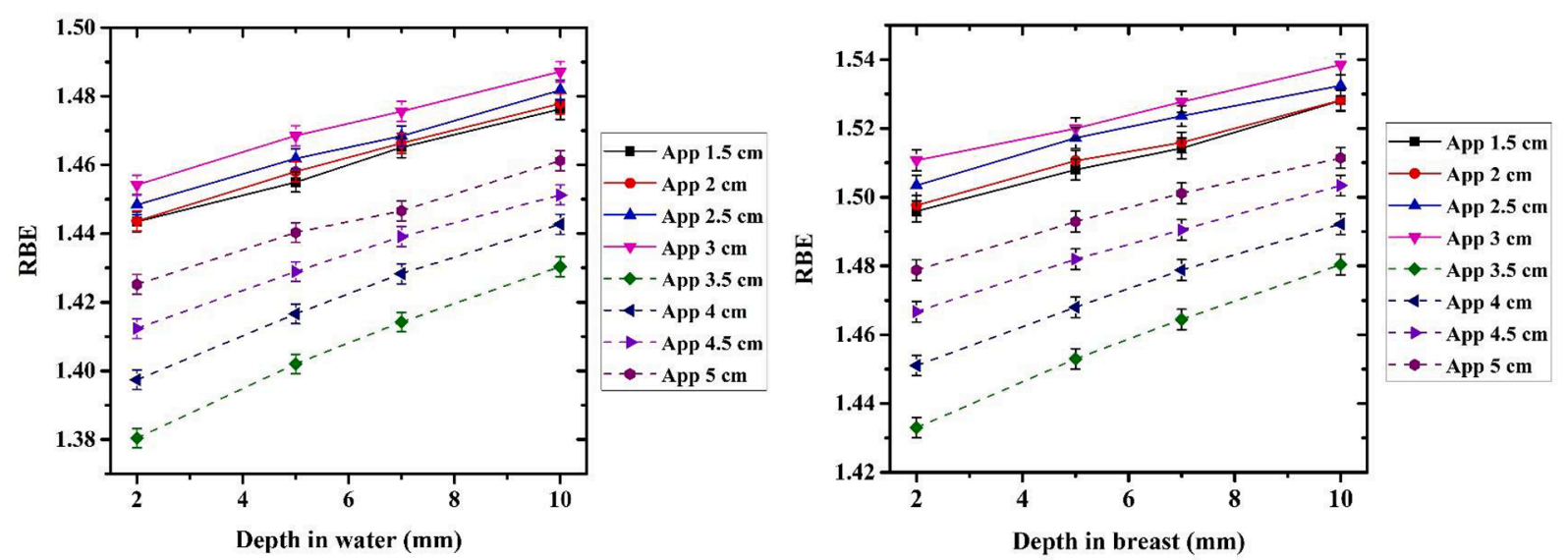

Fig. 10. Variations of RBE value as a function of depth inside the water (left panel) and breast tissue (right panel) for the different spherical applicator diameters. It should be mentioned that the applicators including the Aluminum filter have been shown with solid lines while the filter-free applicators have been illustrated with the dash lines.

\section{References}

[1] Harris EER, Small Jr W. Intraoperative radiotherapy for breast cancer. Front Oncol $2017 ; 7: 317$

[2] Kraus-Tiefenbacher U, Scheda A, Steil V, Hermann B, Kehrer T, Bauer L, et al. Intraoperative radiotherapy (IORT) for breast cancer using the Intrabeam system. Tumori 2005;91:339-45.

[3] Baldassarre G, Belletti B, Mileto M, Massarut S. Mechanism of action of TARGIT. In Pigott k Keshtgar M, Wenz F, editors. Targeted intraoperative radiotherapy in oncology. New York: Springer; 2014. p. 7-12.

[4] Hensley FW. Present state and issues in IORT physics. Radiat Oncol 2017;12:37.

[5] Picot J, Copley V, Colquitt JL, Kalita N, Hartwell D, Bryant J. The INTRABEAM photon radiotherapy system for the adjuvant treatment of early breast cancer: a systematic review and economic evaluation. Health Technol Assess 2015;19: 1-190.

[6] Grobmyer SR, Lightsey JL, Bryant CM, Shaw C, Yeung A, Bhandare N, et al. Low kilovoltage, single-dose intraoperative radiation therapy for breast cancer: results and impact on a multidisciplinary breast cancer program. J Am Coll Surg 2013; 216:617-24.

[7] Vaidya JS, Tobias JS, Baum M, Keshtgar M, Joseph D, Wenz F, et al. Intraoperative radiotherapy for breast cancer. Lancet Oncol 2004;5:165-73.

[8] Kraus-Tiefenbacher U, Biggs P, Vaidya J, Francescatti D. Electronic brachytherapy/ low KV-IORT: physics and techniques. In: Gunderson LL, Calvo FA, Willett CG, Harrison LB, editors. Intraoperative irradiation: Techniques and results. Humana Press; 2011. p. 85-98.

[9] Astor MB, Hilaris BS, Gruerio A, Varricchione T, Smith D. Preclinical studies with the photon radiosurgery system (PRS). Int J Radiat Oncol Biol Phys 2000;47: 809-13.

[10] Tsai JY, Chen FH, Hsieh TY, Hsiao YY. Effects of indirect actions and oxygen on relative biological effectiveness: estimate of DSB induction and conversion induced by gamma rays and helium ions. J Radiat Res 2015;56:691-9.

[11] Nikjoo H, Lindborg L. RBE of low energy electrons and photons. Phys Med Biol 2010;55:65-109.

[12] Goodhead DT. Initial events in the cellular effects of ionizing radiations: clustered damage in DNA. Int J Radiat Biol 1994;65:7-17.

[13] Wallace SS. Enzymatic processing of radiation-induced free radical damage in DNA. Radiat Res 1998;150:60-79.

[14] Chaudhary P, Gwynne D, Doria D, Romagnani L, Maiorino C, Padda H, et al. Effectiveness of laser accelerated ultra-high dose rate protons. In: Bret A, Fajardo M, Westerhof E, Melzer A, Dromey B, Riconda C, editors. Proceedings of the 44th EPS conference on plasma physics; 2017.

[15] Luo WR, Chen FH, Huang RJ, Chen YP, Hsiao YY. Effects of indirect actions and oxygen on relative biological effectiveness: estimate of DSB inductions and conversions induced by therapeutic proton beams. Int J Radiat Biol 2020;96: $187-96$.

[16] Shamsabadi R, Baghani HR, Azadegan B, Mowlavi AA. Monte Carlo based analysis and evaluation of energy spectrum for low kV IORT spherical applicators. Z Med Phys 2020;30:60-9.

[17] Schneider F, Clausen S, Eaton DJ. Quality assurance and commissioning. In: Keshtgar M, Pigott K, Wenz F, editors. Targeted intraoperative radiotherapy in oncology. New York: Springer; 2014. p. 31-5.

[18] White DR, Griffith RV, Wilson IJ. Report 46- Photon, electron, proton and neutron interaction data for body tissues. J ICRU 1992;28.
[19] Beld E, Seevinck PR, Lagendijk JJW, Viergever MA, Moerland MA. Monte Carlo study of the impact of a magnetic field on the dose distribution in MRI-guided HDR brachytherapy using Ir-192. Phys Med Biol 2016;61:6791-807.

[20] Howard M, Beltran C, Sarkaria J, Herman MG. Characterization of relative biological effectiveness for conventional radiation therapy: a comparison of clinical 6 MV X-rays and ${ }^{137}$ Cs. J Radiat Res 2017;58:608-13.

[21] Kellerer AM. Electron spectra and the RBE of X rays. Radiat Res 2002;158:13-22.

[22] Friedland W, Jacob P, Paretzke HG, Merzagora M, Ottolenghi A. Simulation of DNA fragment distributions after irradiation with photons. Radiat Environ Biophys 1999;38:39-47.

[23] Hsiao Y, Stewart RD. Monte Carlo simulation of DNA damage induction by X-ray and selected radioisotopes. Phys Med Biol 2008;53:233-44.

[24] Karger CP, Peschke P. RBE and related modeling in carbon-ion therapy. Phys Med Biol 2017;63:01TR02.

[25] Semenenko VA, Stewart RD. Fast Monte Carlo simulation of DNA damage formed by electrons and light ions. Phys Med Biol 2006;51:1693-706.

[26] Stewart RD, Streitmatter SW, Argento DC, Kirkby C, Goorley JT, Moffitt G, et al Rapid MCNP simulation of DNA double strand break (DSB) relative biological effectiveness (RBE) for photons, neutrons, and light ions. Phys Med Biol 2015;60: 8249-74.

[27] Semenenko VA, Stewart RD. A fast Monte Carlo algorithm to simulate the spectrum of DNA damages formed by ionizing radiation. Radiat Res 2004;161:451-7.

[28] Mavragani IV, Nikiski Z, Kalospyros SA, Georgakilas AG. Ionizing radiation and complex DNA damage: from prediction to detection challenges and biological significance. Cancers (Basel) 2019;11:1789.

[29] Nikjoo H, Bolton CE, Watanabe R, Terrissol M, O'Neill P, Goodhead DT. Modelling of DNA damage induced by energetic electrons ( $100 \mathrm{eV}$ to $100 \mathrm{keV})$. Radiat Prot Dosim 2002;99:77-80.

[30] Stewart RD, Yu VK, Georgakilas AG, Koumenis C, Park JH, Carlson DJ. Effects of radiation quality and oxygen on clustered DNA lesions and cell death. Radiat Res 2011;176:587-602.

[31] Reniers B, Liu D, Rusch T, Verhaegen F. Calculation of relative biological effectiveness of a low-energy electronic brachytherapy source. Phys Med Biol 2008; 53:7125-35.

[32] Ezzati AO, Mahmoud-Pashazadeh A, Studenski MT. Monte Carlo simulation of the RBE of I-131 radiation using DNA damage as biomarker. Australas Phys Eng Sci Med 2017;40(2):395-400.

[33] Stewart RD, Carlson DJ, Butkus MP, Hawkins R, Friedrich T, Scholz M. A comparison of mechanism-inspired models for particle relative biological effectiveness (RBE). Med Phys 2018:925-52.

[34] White SA, Reniers B, Jong EEC, Rusch T, Verhaegen F. A comparison of the relative biological effectiveness of low energy electronic brachytherapy sources in breast tissue: a Monte Carlo study. Phys Med Biol 2016;61:383-99.

[35] Ezzati AO. Relative biological damage in and out of field of 6, 10 and $18 \mathrm{MV}$ clinical photon beams. Eur Phys J Plus 2016;131:1-9.

[36] Vassiliev ON. Electron slowing-down spectra in water for electron and photon sources calculated with the Geant4-DNA code. Phys Med Biol 2012;57:1087-94.

[37] Hill MA. The variation in biological effectiveness of X-ray and gamma rays with energy. Radiat Prot Dosim 2004;112:471-81.

[38] Thomson RM, Tedgren AC, Williamson JF. On the biological basis for competing macroscopic dose descriptors for kilovoltage dosimetry: cellular dosimetry for brachytherapy and diagnostic radiology. Phys Med Biol 2013;58:1123-50. 